\title{
Contribution of MS-based proteomics to the understanding of herpes simplex virus type 1 interaction with host cells
}

\author{
Enrique Santamaría ${ }^{1}$, Virginia Sánchez-Quiles ${ }^{2}$, Joaquín Fernández-Irigoyen $^{1}$ and Fernando Corrales ${ }^{2}$ * \\ 1 Proteomics Unit, Biomedical Research Centre, Navarra Health Service, Pamplona, Spain \\ 2 Proteomics Unit, Centre for Applied Medical Research, University of Navarra, Pamplona, Spain
}

\section{Edited by:}

Kevin Coombs, University of

Manitoba, Canada

Reviewed by:

Kevin Coombs, University of Manitoba, Canada

Roger Lippé, University of Montreal,

Canada

*Correspondence:

Fernando Corrales, Proteomics Unit, Centre for Applied Medical Research, University of Navarra, Pío XII Avenue, 55, 31008 Pamplona, Spain. e-mail: fjcorrales@unav.es

\begin{abstract}
Like other DNA viruses, herpes simplex virus type 1 (HSV-1) replicates and proliferates in host cells continuously modulating the host molecular environment. Following a sophisticated temporal expression pattern, HSV-1 encodes at least 89 multifunctional proteins that interplay with and modify the host cell proteome. During the last decade, advances in mass spectrometry applications coupled to the development of proteomic separation methods have allowed to partially monitor the impact of HSV-1 infection in human cells. In this review, we discuss the current use of different proteome fractionation strategies to define HSV-1 targets in two major application areas: (i) viral-protein interactomics to decipher viral-protein interactions in host cells and (ii) differential quantitative proteomics to analyze the virally induced changes in the cellular proteome. Moreover, we will also discuss the potential application of high-throughput proteomic approaches to study global proteome dynamics and also post-translational modifications in HSV-1-infected cells that will greatly improve our molecular knowledge of HSV-1 infection.
\end{abstract}

Keywords: HSV-1, fractionation, labeling, proteomics, mass spectrometry

\section{INTRODUCTION}

Herpes simplex virus type 1 (HSV-1) is a large, double stranded DNA virus with a genome of $152 \mathrm{kbp}$ that replicates in the nucleus of the host cells and presents a unique genetic flexibility (Taylor et al., 2002). Its gene expression follows a temporal pattern including three stages: immediate early (IE), early (E), and late (L) genes (Clements et al., 1977). The process of infection begins when the virions bind heparin sulfate moieties present on host cell plasma membrane. Within the first $30 \mathrm{~min}$ of infection, the initial attachment triggers a cascade of molecular interactions involving multiple viral and host cell proteins and receptors, leading to penetration of the viral nucleocapsid and tegument proteins into the cytoplasm (Dohner et al., 2006). After penetration, viral capsids and associated tegument proteins interact with dynein and utilize the microtubule network to transit the cytosol to the nuclear envelope, where they dock with nuclear pores and release their genomes into the nucleus. The HSV-1 genome replication starts around $3-4 \mathrm{~h}$ post infection (hpi) reaching maximum efficiency between 8 and 16 hpi (Phelan and Barklie Clements, 1997).

A large amount of information is available about the cellular fate of viral proteins and their constitutive functions in infected cells (Taylor et al., 2002). However, little is known about host effectors involved in viral replication and their virally induced functions, a crucial issue to understand the molecular pathogenesis of HSV-1 in human cells. This biological information is critical to boost novel vaccines and treatments for the broad-spectrum of pathological disorders caused by HSV-1 infection (Al-Dujaili et al., 2011; Chisholm and Lopez, 2011;Rozenberg et al., 2011) and also to promote the development of conditionally replicating HSV-1 vectors expressing cellular genes as anticancer therapeutic agents (Argnani et al., 2011; Shen and Nemunaitis, 2006; Nguyen and Blaho, 2007). In spite of the different genomic approaches carried out to understand the molecular alterations induced by HSV-1 infection in different mammalian cells (Mossman et al., 2001; Higaki et al., 2002; Paludan et al., 2002; Taddeo et al., 2002; Ray and Enquist, 2004; Sun et al., 2004; Clement et al., 2008, 2009; Zeier et al., 2009), changes in mRNA abundance do not always correlate to changes at protein level (Tian et al., 2004). Proteomics, therefore, is expected to provide a more extensive and complementary description of the cellular mechanisms altered upon HSV-1 infection. In this review, we focus on various protein fractionation methods coupled to mass spectrometry (MS) previously used to decipher novel HSV-1 targets. The results discussed here anticipate the future impact of high-throughput proteomic applications in the field of HSV-1 biology.

\section{HSV-1 PROTEIN INTERACTOME}

In the last few years, LC-MS/MS analyses coupled to diverse purification steps has emerged as a powerful technique to elucidate viral proteins interactions with host proteome. In fact, some immediate-early viral-protein interactomes have been partially characterized (Figure 1). Using immunoprecipitation procedures coupled to MS and validation techniques, Fontaine-Rodriguez et al. (2004) identified eIF3 subunits p47 and p116, eIF4G, and poly A binding protein as cellular interactors of ICP27 viral protein in human epidermoid (Hep-2) cells, suggesting that ICP27 may interfere in viral or host mRNA translation. Using a similar approach, Taylor and Knipe (2004) analyzed the interaction partners of ICP8 in Hep-2 cells identifying over 50 cellular proteins (and also some viral proteins) that coimmunoprecipitate or localize with ICP8 in replication compartments. This potential 


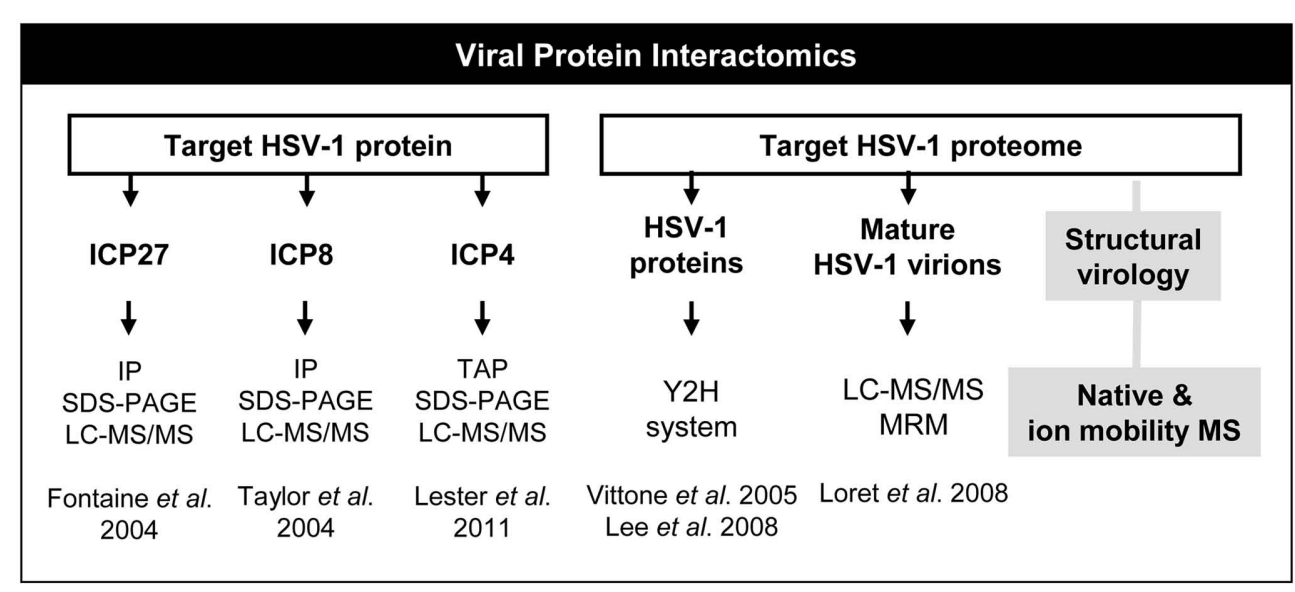

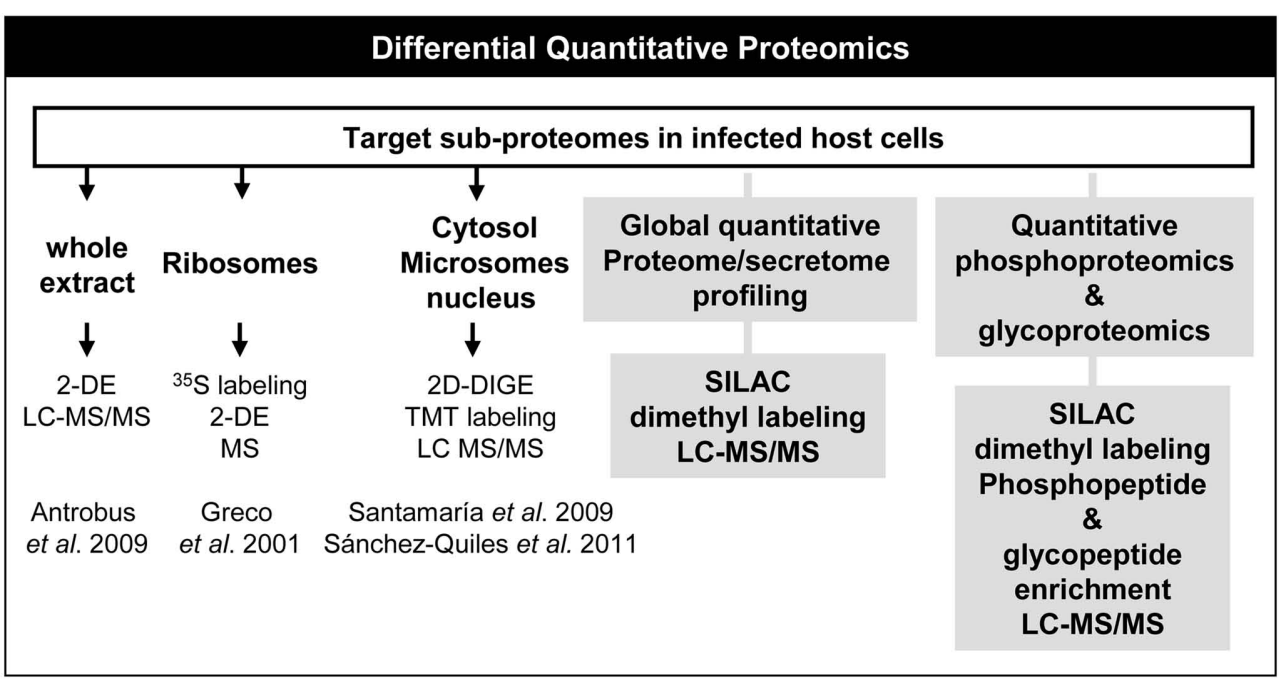

FIGURE 1 | Current and future applications of mass spectrometry to HSV-1 infection. Schematic representation of how proteomic technologies have been used to detect and identify HSV-1 targets in protein interactome experiments and also in differential quantitative protein expression studies. Gray boxes refer to alternative unexplored approaches that can be used to obtain more detailed information about the composition of HSV-1 particle (structural virology) and to analyze large-scale proteome perturbations and signaling pathways in host cells upon HSV-1 infection (Global and site specific quantitative glyco- and phosphoproteomics). IP, immunoprecipitation; LC-MS/MS, liquid chromatography coupled to tandem mass spectrometry; TAP, tandem affinity purification; $\mathrm{Y} 2 \mathrm{H}$, yeast two-hybrid system; MRM, multiple reaction monitoring; MS, mass spectrometry; 2-DE, two-dimensional electrophoresis; 2D-DIGE, two-dimensional fluorescence difference gel electrophoresis; TMT, Tandem mass tag isobaric labeling; SILAC, stable isotope labeling with amino acids in cell culture. interactome was involved in different molecular function such as transcription, replication and recombination, chromatin remodeling, and RNA splicing (Taylor and Knipe, 2004). More recently, Lester and Deluca (2011) have isolated the ICP4-containing complexes from HeLa-infected cells using tandem affinity purification (TAP-ICP4) and protein fractionation by $1 \mathrm{D}$ gel SDS-PAGE, identifying more than 40 cellular proteins present in theses complexes by LC-MS/MS from which 11 and 4 proteins were components of the transcription factor TFIID and the mediator complex respectively. In this case, proteomic data were functionally validated by immunofluorescence and chromatin immunoprecipitation experiments showing that TFIID and mediator are recruited to viral promoters as a function of ICP4 during infection (Lester and Deluca, 2011). On the other hand, more than 40 binary interactions have been detected between capsid, envelope, and tegument viral proteins using protein-protein interaction yeast two-hybrid system (Bernhard et al., 2005; Vittone et al., 2005; Lee et al., 2008). Different proteome composition of purified mature extracellular viruses has been also characterized by MS-based proteomics technology. The carefully developed workflow used by Loret et al. (2008) allowed the identification of 8 viral capsid proteins, 23 potential tegument proteins, and 13 viral glycoproteins. In this workflow, a multiple reaction monitoring (MRM) approach was used to detect specific viral glycoproteins in mature virion preparations. Interestingly, 49 different host proteins mainly involved in transport, cytoskeleton, and nucleotide binding were also accurately identified. Although it is necessary to functionally validate the presence of these host proteins in the extracellular virion structure, 
these data clearly demonstrate that HSV-1 tegumentation may be considered as a highly dynamic process.

\section{PROTEOMIC PROFILING OF HSV-1-INFECTED CELLS}

Comparative proteomic strategies have been also used in HSV1 research to elucidate host proteome modifications during the early stages of HSV-1 infection (Figure 1). One of the most popular methods for protein separation is 2D-electrophoresis (2-DE). Based on two independent biochemical characteristics of proteins, it combines isoelectric focusing, which separates proteins according to their isoelectric point (pI), and SDS-PAGE, which separates them further according to their molecular mass (Westermeier and Gorg, 2011). Using ${ }^{35} \mathrm{~S}$ labeling and 2-DE, Greco et al. (2000) demonstrated that although a strong shutoff in protein synthesis is induced in HSV-1 infection, the synthesis of some acidic ribosomal proteins is sustained or even induced early in infection. On the other hand, Antrobus et al. identified more than 100 protein species differentially expressed between non-infected and infected Hep-2 cells at 6 hpi. They used conventional 2-DE for protein separation and MS for protein identification. The host proteins identified in this study participate in a plethora of biological process and some of them were validated by western-blot analysis also in human diploid fibroblast (HF) cells (Antrobus et al., 2009).

Advanced prefractionation strategies in combination with MS, have provided powerful means to enrich and analyze organellespecific subproteomes, obtaining valuable information that greatly expand our knowledge of the molecular mechanisms altered during HSV-1 infection. With the aim of detecting non-ribosomal proteins associated to the ribosome that may contribute to the HSV-1-induced translational control, Greco et al. extracted basic proteins from the ribosomal fraction from mock-infected and HSV-1-HeLa-infected cells. Using ${ }^{35}$ S, 2-DE, N-terminal sequencing, and MS, viral proteins such as VP19C and VP26, and host poly(A) binding protein 1 were identified associated to ribosomes with different kinetics upon HSV-1 infection (Greco et al., 2001). More recently, we have used complementary protein and peptide labeling strategies to understand the dynamics of the human hepatoma cell proteome behavior upon HSV-1 infection. To increase the efficiency of proteomic analysis, Santamaría et al. fractionated mock-infected and HuH7-infected cells to obtain cytosolic and microsomal proteomes independently, at different early time points of HSV-1 infection. After validating the efficiency of the enrichment procedure, proteome dynamics was analyzed in both subcellular fractions by two-dimensional fluorescence difference gel electrophoresis (2D-DIGE). This approach is based on direct labeling of lysine groups on proteins with cyanine CyDye DIGE Fluor minimal dyes before isoelectric focusing, enabling the labeling of two to three samples with different dyes and electrophoresis of all the samples on the same 2D gel, minimizing spot pattern variability and the number of gels in an experiment (Tannu and Hemby, 2006). Using this approach, 16 unique deregulated host protein species mainly involved in apoptosis, signal transduction, and endoplasmic reticulum associated degradation pathway were identified in $\mathrm{HuH} 7$-infected cells by LC-MS/MS analyses (Santamaria et al., 2009). Interestingly, three of these proteins (FK506-binding protein 4, hnRNPK and eukaryotic translation initiation factor 3 subunit 2) were also deregulated early upon infection in Hep-2 cells (Antrobus et al., 2009). These coincident proteins suggest that cellular mechanisms involved in microtubule dynamics and mRNA processing are common in infected cells with different origin indicating the importance of these targets for HSV-1 infection. Santamaria et al. (2009) demonstrated the activation of mitochondrial apoptotic and different canonical signaling pathways identifying PP2A as a pivotal target that may mediate the transduction of cell death signals and promote cell survival pathway arrest in $\mathrm{HuH} 7$ cells. In the near future, it would be interesting to study the specific role of PP2A in different kind of cells infected with other HSV-1 strains to obtain robust conclusions. Moreover, deciphering the potential interactome of PP2A together with phosphoproteomewide analysis of infected cells would increase our knowledge about the specific role of phosphorylation in HSV-1-infected cells.

Taking into account that HSV-1 replicates in the nucleus of the host cells, Sanchez-Quiles et al. (2011) have combined 2DDIGE and tandem mass tag isobaric labeling (TMT) techniques to analyze nuclear proteome alterations in HuH7-infected cells. TMT is a liquid-based method that labels samples at the peptide level (Dayon et al., 2008). While DIGE separates only soluble proteins included in a $\mathrm{pH}$ range of 3-11 and a determined molecular weight, TMT can identify proteins outside these ranges. Moreover, DIGE is able to detect differential post-translationally modified proteins as well as different isoforms by resolving spots at different pI and molecular weight. However, these isoforms may not be distinguished with TMT approach, since labeling is completed at the peptide level and most peptide sequences are identical among a group of isoforms. In addition, proteins with extreme isoelectric points, large molecular weights, low solubility (hydrophobic proteins) and low copy numbers are poorly represented in 2D-DIGE experiments. The application of two different proteomic strategies was particularly useful in obtaining complementary information, identifying 62 differential components of the nuclear proteome of Huh7 hepatoma cells that may regulate host-virus interaction, cell cycle regulation, and RNA homeostasis upon infection (Sanchez-Quiles et al., 2011). In particular, Lamin A-C, also differentially expressed in Hep-2 infected cells (Antrobus et al., 2009), showed a complex differential isoform pattern upon HSV-1 infection, suggesting regulatory mechanisms based on post-translational modification events. Interestingly, 32 host proteins were enriched in infected nuclei. One of these early up-regulated proteins was protein quaking $(\mathrm{QKI})$, involved in the regulation of mRNA stability, nuclear retention, and RNA transport (Galarneau and Richard, 2005). Using immunofluorescence assays, Sánchez-Quiles et al. have demonstrated that QKI-5 suffers a time-dependent redistribution in infected cells, shuttling from the nucleus to the cytosol upon HSV-1 infection. Interestingly, QKI depletion by knockdown strategy induces a clear delay in viral-protein synthesis, reducing the viral yield in infected cells (Sanchez-Quiles et al., 2011). Although the viral necessity of maintaining high expression levels of QKI during the infection should be demonstrated as a possible global mechanism of HSV-1 interference with host cells, these data suggest that QKI may be a potential target in the development of antiviral therapy. 
All differential proteins provided by proteomic studies have undoubtedly extended our knowledge about HSV-1 infection at the molecular level. However, the protein repertoires reported are barely coincident. This variability is likely associated with specific biological properties of the samples analyzed (different cell lines, HSV-1 strains, and time point of infection used) and the heterogeneity of the analytical procedures used (fractionation methods to isolate specific organelles, and the use of gel-based or gel free proteomic workflows). Although differential proteomics has contributed to a better understanding about the specific proteome changes that occur early in infection, additional research is needed to elucidate whether these differentially expressed proteins are cellular targets used for HSV1 to modulate the host proteome or are part of molecular events orchestrated by the cell to limit viral replication and propagation.

\section{FUTURE DIRECTIONS}

Although 2D-electrophoresis has been the technique of choice to monitor changes at protein level in HSV-1-infected cells, this approach presents some inherent disadvantages such as many protein spots are likely comprised of multiple proteins, poor spot resolution at higher pI values, and the difficulty in electrophoresing large and hydrophobic proteins in the first dimension separation. However, the rapid advances in high-resolution MS-based proteomics strategies may facilitate the global analysis of protein interacting partners of HSV-1 virion proteomes (Bailer and Haas, 2009), host protein components in HSV-1-infected secretome (Higa et al., 2008; Deng et al., 2010; Lietzen et al., 2011), and

\section{REFERENCES}

Al-Dujaili, L. J., Clerkin, P. P., Clement,

C., Mcferrin, H. E., Bhattacharjee, P.

S., Varnell, E. D., Kaufman, H. E., and Hill, J. M. (2011) Ocular herpes simplex virus: how are latency, reactivation, recurrent disease and therapy interrelated? Future Microbiol. 6, 877-907.

Antrobus, R., Grant, K., Gangadharan, B., Chittenden, D., Everett, R. D., Zitzmann, N., and Boutell, C. (2009). Proteomic analysis of cells in the early stages of herpes simplex virus type- 1 infection reveals widespread changes in the host cell proteome. Proteomics 9, 3913-3927.

Argnani, R., Marconi, P., Volpi, I., Bolanos, E., Carro, E., Ried, C., Santamaria, E., Pourchet, A., Epstein, A. L., Brocker, T., Corrales, F. J., Manservigi, R., Goicoechea, I., Foschini, M., and Hernandez-Alcoceba, R. (2011). Characterization of herpes simplex virus 1 strains as platforms for the development of oncolytic viruses against liver cancer. Liver Int. 31, 1542-1553.

Bailer, S. M., and Haas, J. (2009). Connecting viral with cellular

also the detection, identification, and quantitation of thousands of novel HSV-1 targets in host cells to finally obtain an integrative view of proteome modifications during the viral cycle. In the near future, alternative proteomics methods such as SILAC (Ong and Mann, 2006; Harsha et al., 2008) or stable isotope dimethyl labeling (Boersema et al., 2009) will lead to more comprehensive and time-dependent analysis of HSV-1-induced changes in host proteomes. Nowadays, these high-throughput approaches have been established and constantly optimized in order to improve our understanding of phosphorylation events. Large-scale proteomic studies based on SILAC in combination with strong cation exchange (SCX) and titanium dioxide $\left(\mathrm{TiO}_{2}\right)$ for phosphopeptide enrichment (Macek et al., 2009) would greatly contribute to the study of phosphorylation dynamics in HSV-1-infected cells. Using this shotgun approach, unexpected novel phosphorylation sites could be discovered in HSV-1 proteome, increasing our biological knowledge about the activation/deactivation status of multiple host signaling pathways during the infection. Moreover, the combination of immunoaffinity chromatography and MS-based quantitative MS (Pan et al., 2011), will be a valuable approach to characterize the glycoproteome of HSV-1-infected cells and also to map the specific changes in glycosylation patterns of HSV-1 surface proteins during the viral cycle. Finally, the application of native and ion-mobility MS in the near future (Uetrecht et al., 2011) will provide useful information about viral-protein and protein-complex structure, conformation, stoichiometry of an assembly, binding affinities, and the topology of a viral-protein complex (Uetrecht and Heck, 2011) increasing our knowledge on structural HSV-1 biology.

between naive and HSV-1 latent rabbit trigeminal ganglia. Mol. Vis. 14, 1209-1221.

Clements, J. B., Watson, R. J., and Wilkie, N. M. (1977). Temporal regulation of herpes simplex virus type 1 transcription: location of transcripts on the viral genome. Cell 12, 275-285.

Dayon, L., Hainard, A., Licker, V., Turck, N., Kuhn, K., Hochstrasser, D. F., Burkhard, P. R., and Sanchez, J. C. (2008). Relative quantification of proteins in human cerebrospinal fluids by MS/MS using 6-plex isobaric tags. Anal. Chem. 80, 2921-2931.

Deng, W., De Hoog, C. L., Yu, H. B., Li, Y., Croxen, M. A., Thomas, N. A., Puente, J. L., Foster, L. J., and Finlay, B. B. A. (2010). Comprehensive proteomic analysis of the type III secretome of Citrobacter rodentium. J. Biol. Chem. 285, 6790-6800.

Dohner, K., Radtke, K., Schmidt, S., and Sodeik, B. (2006). Eclipse phase of herpes simplex virus type 1 infection: efficient dynein-mediated capsid transport without the small capsid protein VP26. J. Virol. 80, 8211-8224.
Fontaine-Rodriguez, E. C., Taylor, T. J., Olesky, M., and Knipe, D. M. (2004). Proteomics of herpes simplex virus infected cell protein 27: association with translation initiation factors. Virology 330, 487-492.

Galarneau, A., and Richard, S. (2005). Target RNA motif and target mRNAs of the quaking STAR protein. Nat. Struct. Mol. Biol. 12, 691-698.

Greco, A., Bausch, N., Coute, Y., and Diaz, J. J. (2000). Characterization by two-dimensional gel electrophoresis of host proteins whose synthesis is sustained or stimulated during the course of herpes simplex virus type 1 infection. Electrophoresis 21, 2522-2530.

Greco, A., Bienvenut, W., Sanchez, J. C., Kindbeiter, K., Hochstrasser, D., Madjar, J. J., and Diaz, J. J. (2001). Identification of ribosomeassociated viral and cellular basic proteins during the course of infection with herpes simplex virus type 1. Proteomics 1, 545-549.

Clement, C., Popp, M. P., Bloom, D. C., Schultz, G., Liu, L., Neumann, D. M., Bhattacharjee, P. S., and Hill, J. M. (2008). Microarray analysis of host gene expression for comparison
Harsha, H. C., Molina, H., and Pandey, A. (2008). Quantitative proteomics using stable isotope labeling with amino acids in cell culture. Nat. Protoc. 3, 505-516. 
Higa, L. M., Caruso, M. B., Canellas, F., Soares, M. R., Oliveira-Carvalho, A. L., Chapeaurouge, D. A., Almeida, P. M., Perales, J., Zingali, R. B., and Da Poian, A. T. (2008). Secretome of HepG2 cells infected with dengue virus: implications for pathogenesis. Biochim. Biophys. Acta 1784, 1607-1616.

Higaki, S., Gebhardt, B. M., Lukiw, W. J., Thompson, H. W., and Hill, J. M. (2002). Effect of immunosuppression on gene expression in the HSV1 latently infected mouse trigeminal ganglion. Invest. Ophthalmol. Vis. Sci. 43, 1862-1869.

Lee, J. H., Vittone, V., Diefenbach, E., Cunningham, A. L., and Diefenbach, R. J. (2008). Identification of structural protein-protein interactions of herpes simplex virus type 1. Virology 378, 347-354.

Lester, J. T., and Deluca, N. A. (2011). Herpes simplex virus 1 ICP4 forms complexes with TFIID and mediator in virus-infected cells. J. Virol. 85 , 5733-5744.

Lietzen, N., Ohman, T., Rintahaka, J., Julkunen, I., Aittokallio, T., Matikainen, S., and Nyman, T. A. (2011). Quantitative subcellular proteome and secretome profiling of influenza A virus-infected human primary macrophages. PLoS Pathog. 7, el001340. doi:10.1371/journal.ppat.1001340

Loret, S., Guay, G., and Lippe, R. (2008). Comprehensive characterization of extracellular herpes simplex virus type 1 virions. J. Virol. 82, 8605-8618.

Macek, B., Mann, M., and Olsen, J. V. (2009). Global and sitespecific quantitative phosphoproteomics: principles and applications. Annu. Rev. Pharmacol. Toxicol. 49, 199-221.

Mossman, K. L., Macgregor, P. F., Rozmus, J. J., Goryachev, A. B., Edwards, A. M., and Smiley, J. R. (2001). Herpes simplex virus triggers and then disarms a host antiviral response. $J$. Virol. 75, 750-758.

Nguyen, M. L., and Blaho, J. A. (2007). Apoptosis during herpes simplex virus infection. Adv. Virus Res. 69, 67-97.

Ong, S. E., and Mann, M. (2006). A practical recipe for stable isotope labeling by amino acids in cell culture (SILAC). Nat. Protoc. 1, 2650-2660.

Paludan, S. R., Melchjorsen, J., Malmgaard, L., and Mogensen, S. C. (2002). Expression of genes for cytokines and cytokine-related functions in leukocytes infected with herpes simplex virus: comparison between resistant and susceptible mouse strains. Eur. Cytokine Netw. 13, 306-316.

Pan, S., Chen, R., Aebersold, R., and Brentnall, T. A. (2011). Mass spectrometry based glycoproteomics from a proteomics perspective. $\mathrm{Mol}$. Cell Proteomics 10, R110.003251.

Phelan, A., and Barklie Clements, J. (1997). Functional domains within the nucleus of a cell infected with HSV-1. Rev. Med. Virol. 7, 229-237.

Ray, N., and Enquist, L. W. (2004). Transcriptional response of a common permissive cell type to infection by two diverse alphaherpesviruses. $J$. Virol. 78, 3489-3501.

Rozenberg, F., Deback, C., and Agut, H. (2011). Herpes simplex encephalitis: from virus to therapy. Infect. Disord. Drug Targets 11, 235-250.

Sanchez-Quiles, V., Mora, M. I., Segura, V., Greco, A., Epstein, A. L., Foschini, M. G., Dayon, L., Sanchez, J. C., Prieto, J., Corrales, F. J., and Santamaria, E. (2011). HSV-1 Cgal+ infection promotes quaking RNA binding protein production and induces nuclear-cytoplasmic shuttling of quaking I-5 isoform in human hepatoma cells. Mol. Cell Proteomics 10, M111.009126.

Santamaria, E., Mora, M. I., Potel, C., Fernandez-Irigoyen, J., CarroRoldan, E., Hernandez-Alcoceba, R., Prieto, J., Epstein, A. L., and Corrales, F. J. (2009). Identification of replication-competent HSV1 Cgal+ strain signaling targets in human hepatoma cells by functional organelle proteomics. Mol. Cell Proteomics 8, 805-815.
Shen, Y., and Nemunaitis, J. (2006). Herpes simplex virus 1 (HSV-1) for cancer treatment. Cancer Gene Ther. 13 975-992.

Sun, A., Devi-Rao, G. V., Rice, M. K., Gary, L. W., Bloom, D. C., SandriGoldin, R. M., Ghazal, P., and Wagner, E. K. (2004). Immediate-early expression of the herpes simplex virus type 1 ICP27 transcript is not critical for efficient replication in vitro or in vivo. J. Virol. 78, 10470-10478.

Taddeo, B., Esclatine, A., and Roizman, B. (2002). The patterns of accumulation of cellular RNAs in cells infected with a wild-type and a mutant herpes simplex virus 1 lacking the virion host shutoff gene. Proc. Natl. Acad. Sci. U.S.A. 99, 17031-17036.

Tannu, N. S., and Hemby, S. E. (2006). Two-dimensional fluorescence difference gel electrophoresis for comparative proteomics profiling. Nat. Protoc. 1, 1732-1742.

Taylor, T. J., Brockman, M. A., Mcnamee, E. E., and Knipe, D. M. (2002). Herpes simplex virus. Front. Biosci. 7 , d752-d764.

Taylor, T. J., and Knipe, D. M. (2004). Proteomics of herpes simplex virus replication compartments: association of cellular DNA replication, repair, recombination, and chromatin remodeling proteins with ICP8. J. Virol. 78, 5856-5866.

Tian, Q., Stepaniants, S. B., Mao, M. Weng, L., Feetham, M. C., Doyle, M. J., Yi, E. C., Dai, H., Thorsson, V., Eng, J., Goodlett, D., Berger, J. P., Gunter, B., Linseley, P. S., Stoughton, R. B. Aebersold, R., Collins, S. J., Hanlon, W. A., and Hood, L. E. (2004). Integrated genomic and proteomic analyses of gene expression in mammalian cells. Mol. Cell Proteomics 3 , 960-969.

Uetrecht, C., Barbu, I. M., Shoemaker, G. K., Van Duijn, E., and Heck, A. J. (2011). Interrogating viral capsid assembly with ion mobilitymass spectrometry. Nat. Chem. 3, 126-132.
Uetrecht, C., and Heck, A. J. (2011). Modern biomolecular mass spectrometry and its role in studying virus structure, dynamics, and assembly. Angew. Chem. Int. Ed. Engl. 50, 8248-8262.

Vittone, V., Diefenbach, E., Triffett, D., Douglas, M. W., Cunningham, A. L., and Diefenbach, R. J. (2005). Determination of interactions between tegument proteins of herpes simplex virus type 1. J. Virol. 79, 9566-9571.

Westermeier, R., and Gorg, A. (2011). Two-dimensional electrophoresis in proteomics. Methods Biochem. Anal. 54, 411-439.

Zeier, Z., Aguilar, J. S., Lopez, C. M., Devi-Rao, G. B., Watson, Z. L., Baker, H. V., Wagner, E. K., and Bloom, D. C. (2009). A limited innate immune response is induced by a replicationdefective herpes simplex virus vector following delivery to the murine central nervous system. J. Neurovirol. 15, 411-424.

Conflict of Interest Statement: The authors declare that the research was conducted in the absence of any commercial or financial relationships that could be construed as a potential conflict of interest.

Received: 02 February 2012; accepted: 02 March 2012; published online: 20 March 2012.

Citation: Santamaría E, Sánchez-Quiles $V$, Fernández-Irigoyen $J$ and Corrales $F$ (2012) Contribution of MS-based proteomics to the understanding of herpes simplex virus type 1 interaction with host cells. Front. Microbio. 3:107. doi: 10.3389/fmicb.2012.00107

This article was submitted to Frontiers in Virology, a specialty of Frontiers in Microbiology.

Copyright () 2012 Santamaría, SánchezQuiles, Fernández-Irigoyen and Corrales. This is an open-access article distributed under the terms of the Creative Commons Attribution Non Commercial License, which permits noncommercial use, distribution, and reproduction in other forums, provided the original authors and source are credited. 